\title{
Correlation between the Modified Systemic Lupus Erythematosus Disease Activity Index 2000 and the European Consensus Lupus Activity Measurement in juvenile systemic lupus erythematosus
}

\author{
JO Sato ${ }^{1}$, JE Corrente ${ }^{2}$ and C Saad-Magalhães ${ }^{1}$ \\ ${ }^{1}$ Departamento de Pediatria, Faculdade de Medicina de Botucatu, UNESP_Universidade Estadual Paulista, São Paulo, Brazil; and \\ ${ }^{2}$ Departamento de Bioestatística, Instituto de Biociências, Campus de Botucatu, UNESP-Universidade Estadual Paulista, São Paulo, Brazil
}

\begin{abstract}
Objective: The objective of this study was to assess Modified Systemic Lupus Erythematosus Disease Activity Index 2000 (SLEDAI-2K) and European Consensus Lupus Activity Measurement (ECLAM) disease activity correlation in addition to their respective correlation to Pediatric Systemic Lupus International Collaborative Clinics/American College of Rheumatology (SLICC/ACR) Damage Index (Ped-SDI), in juvenile systemic lupus erythematosus (JSLE). Methods: The activity indices were scored retrospectively and summarized by adjusted means during follow-up. The Ped-SDI was scored during the last visit for those with more than six months follow-up. Pearson correlation between the Modified SLEDAI-2K and ECLAM, as well as Spearman correlations between the Modified SLEDAI-2K, ECLAM, and Ped-SDI were calculated. The receiver operating characteristic (ROC) curve was calculated for both activity indices discriminating damage measured by Ped-SDI. Results: Thirty-seven patients with mean age at diagnosis $11 \pm 2.9$ years and mean follow-up time $3.2 \pm 2.4$ years were studied. The Modified SLEDAI-2K and ECLAM adjusted means were highly correlated $(r=0.78, p<0.001)$. Similarly, Spearman correlation between the activity indices was also high $\left(r_{\mathrm{s}}>0.7, p<0.001\right)$, but Modified SLEDAI-2K and ECLAM correlation with Ped-SDI was only moderate. ROC analysis discriminant performance for both activity indices resulted in area under curve (AUC) of 0.74 and 0.73 for Modified SLEDAI-2K and ECLAM, respectively. Conclusion: The high correlation found between the Modified SLEDAI-2K and ECLAM adjusted means indicated that both tools can be equally useful for longitudinal estimates of JSLE activity. Lupus (2016) 25, 1479-1484.
\end{abstract}

Key words: Activity; childhood-onset systemic lupus erythematosus; damage; ECLAM; juvenile systemic lupus erythematosus; Modified SLEDAI-2K

\section{Introduction}

Activity and damage indices are commonly used to assess morbidity in juvenile systemic lupus erythematosus (JSLE). Valid tools have been developed for JSLE. ${ }^{1,2}$ The most used are the Systemic Lupus Erythematosus Disease Activity Index (SLEDAI), the British Isles Lupus Assessment Group (BILAG), and the Systemic Lupus Activity Measure (SLAM). The Modified SLEDAI-2K is a variant of the original SLEDAI that suppresses

Correspondence to: Juliana de Oliveira Sato, Departamento de Pediatria, Faculdade de Medicina de Botucatu, Universidade Estadual Paulista (UNESP), 18603-970 Botucatu, São Paulo, Brazil. Email: julianasato@fmb.unesp.br

Received 9 September 2015; accepted 20 April 2016
anti-dsDNA and complement. ${ }^{3}$ It has not been previously tested in JSLE. On the other hand, SLEDAI, BILAG, and SLAM have shown equivalent responsiveness in pediatric patients, but to date there is no gold standard for disease activity measurement in JSLE. ${ }^{2,4,5}$

We have previously addressed scoring activity to establish disease patterns related to damage accrual. ${ }^{6}$ Since there is no consensus regarding what is the best disease activity index, we believe it could be valuable to analyze the correlation between different disease activity tools scored over time in a longitudinal approach. We chose the Modified SLEDAI-2K and the ECLAM because both spare some laboratory tests, which is convenient for daily practice or retrospective review. Both are global and multidimensional indices with 
1480

weighted items. Previous studies comparing both tools suggested that ECLAM would be preferable over the SLEDAI due to better psychometric properties, including good construct validity, discriminant capacity to predict damage, and responsiveness, although there are some criticisms due to lack of definitions for recall time, symptoms glossary, and investigations. SLEDAI has been preferred as easy and quick to complete. ${ }^{2,4,5}$

The aims were assessing the correlation between two activity indices, the Modified SLEDAI-2K and the ECLAM, as well as their respective correlation with the Pediatric SLICC/ACR Damage Index $(\text { Ped-SDI })^{7}$ in JSLE patients.

\section{Patients and methods}

\section{Subjects}

All consecutive patients less than 18 years old diagnosed with JSLE classified according to the 1997 American College of Rheumatology (ACR) criteria, ${ }^{8}$ from 1992 to 2012, were included. Discontinued follow-up, missing notes, immunodeficiency, or other autoimmune disease overlap were exclusion criteria. The research protocol was approved by the institution's ethics committee (no. 460/2009) and conducted in accordance to the Helsinki Declaration.

\section{Procedures}

A secondary analysis of activity and damage indices in a JSLE series was carried out. The protocol was primarily designed to assess lupus activity over time in order to establish its relationship to damage accrual, death, and growth failure. ${ }^{6}$ The present analysis was conducted to further explore the correlation between these two activity indices, the Modified SLEDAI-2K and the ECLAM, and their respective correlation to the Ped-SDI. ${ }^{6}$ Data were recorded in a standardized case-report form for all JSLE patients. Case-notes review of scheduled outpatient visits or hospital admissions were thoroughly examined for clinical complaints, physical examination, and abnormal laboratory tests due to lupus involvement.

\section{Assessment of disease activity}

Two disease activity indices, Modified SLEDAI-2K and ECLAM, were retrospectively scored at each patient visit. Briefly, the Modified SLEDAI- $2 \mathrm{~K}^{3}$ is a version of the SLEDAI-2K that excludes two items from the original tool, the complement levels and anti-dsDNA titers, maintaining 16 clinical manifestations and four laboratory tests (white blood cell count, platelets count, urinalysis, and 24-hour proteinuria), making 22 items evaluating nine system domains. The item is scored if the descriptor is present at the time of visit or in the past ten days. Items are weighted according to the systems involved. Scores range from 0 to 101, 101 being the maximum activity score.

ECLAM includes 32 items evaluating 12 systems, including one item for the constitutional domain. Likewise, items are weighted according to the systems involved. Twenty-two clinical manifestations and ten laboratory tests (hemoglobin, white blood cell count, platelet count, ESR, urinalysis, 24-hour proteinuria, serum creatinine or creatinine clearance, and $\mathrm{C} 3$ or CH50 levels) are scored if the descriptor has been observed during the last 30 days or since the last visit. Scores range from 0 to 10,10 being the maximum activity score. ${ }^{9}$ Specific rules for ECLAM scoring and rounding procedures were followed. Two points should be added to the final score if only one system is involved: if the final score is not an integer number, round off to the lower integer number for values $\leq 6$ and to the higher integer number if $>6$; and, last, if the final score is $>10$, round off to $10 .^{5}$ The ECLAM and the original version of the SLEDAI were previously validated for JSLE. ${ }^{4,5}$ Both SLEDAI and ECLAM can be retrospectively scored. ${ }^{10,11}$

\section{Assessment of disease damage}

Only patients followed over six months or more had damage scored by the Ped-SDI. ${ }^{7}$ Scores range from 0 to 49, with 0 being the absence of damage. A glossary defines item scoring in several organs and tissues. This is the only assessment tool for damage scoring in pediatric lupus patients. Besides the 12 domains of organ damage, scoring $1-3$, additional domains for growth and pubertal development are added for pediatric patients.

\section{Statistical analysis}

Qualitative variables were presented by absolute frequency and percentage. Quantitative variables were presented by mean and standard deviation (SD). Since the time interval between visits was variable, longitudinal analysis of disease activity was conducted according to the method proposed by Ibañez et al., ${ }^{12}$ calculating the adjusted means for the Modified SLEDAI-2K and ECLAM, as follows: (1) calculate the area under the curve (AUC) between two visits by multiplying the time between the two visits by the mean activity score; (2) sum 
the AUC for all visit intervals; (3) divide the result by the follow-up time. This approach resulted in a weighted average of the respective disease activity index for each of the subjects throughout followup. Figure 1 shows one visit-to-visit Modified SLEDAI-2K and ECLAM score, and the respective adjusted means.

A simple linear regression analysis between the adjusted means of the Modified SLEDAI-2K and ECLAM was performed. The linear equation was calculated with its intercept (a), slope (b), coefficient of determination $\left(R^{2}\right)$, and Pearson correlation coefficient $(r)$. The $r$ values ranged from -1 to +1 . Considering the absolute values, Pearson correlation coefficient $(r) \leq 0.3$ was considered poor correlation, between 0.3 and 0.7 moderate correlation, and $\geq 0.7$ high correlation.

The Spearman correlation coefficient $\left(r_{\mathrm{s}}\right)$ between the activity indices, at disease onset and throughout disease course, was calculated. The same coefficient was also calculated between each of the activity indices and the damage index. $r_{\mathrm{s}}$ values range from -1 to +1 . The same thresholds used for the Pearson correlation was also used for the Spearman correlation interpretation.

The receiver operating characteristic (ROC) analysis was conducted to verify the performance of both the Modified SLEDAI-2K and ECLAM in discriminating damage by the Ped-SDI. Results were expressed by AUC, sensitivity, specificity, and cut-off points for the Modified SLEDAI-2K and ECLAM.

SAS for Windows version 9.2, SPSS version 19, and STATISTICA for Windows version 10.0 software were used in all analyses, considering a significance level of $5 \%$ for all tests, with corresponding $p$-value, two-tailed.

\section{Results}

\section{Demographics and clinical characteristics}

Thirty seven JSLE patients were included in this study. Of those, $30(81.1 \%)$ were female. Mean age at diagnosis was $11( \pm 2.9)$ years and mean follow-up duration was $3.2( \pm 2.4)$ years. Treatment results

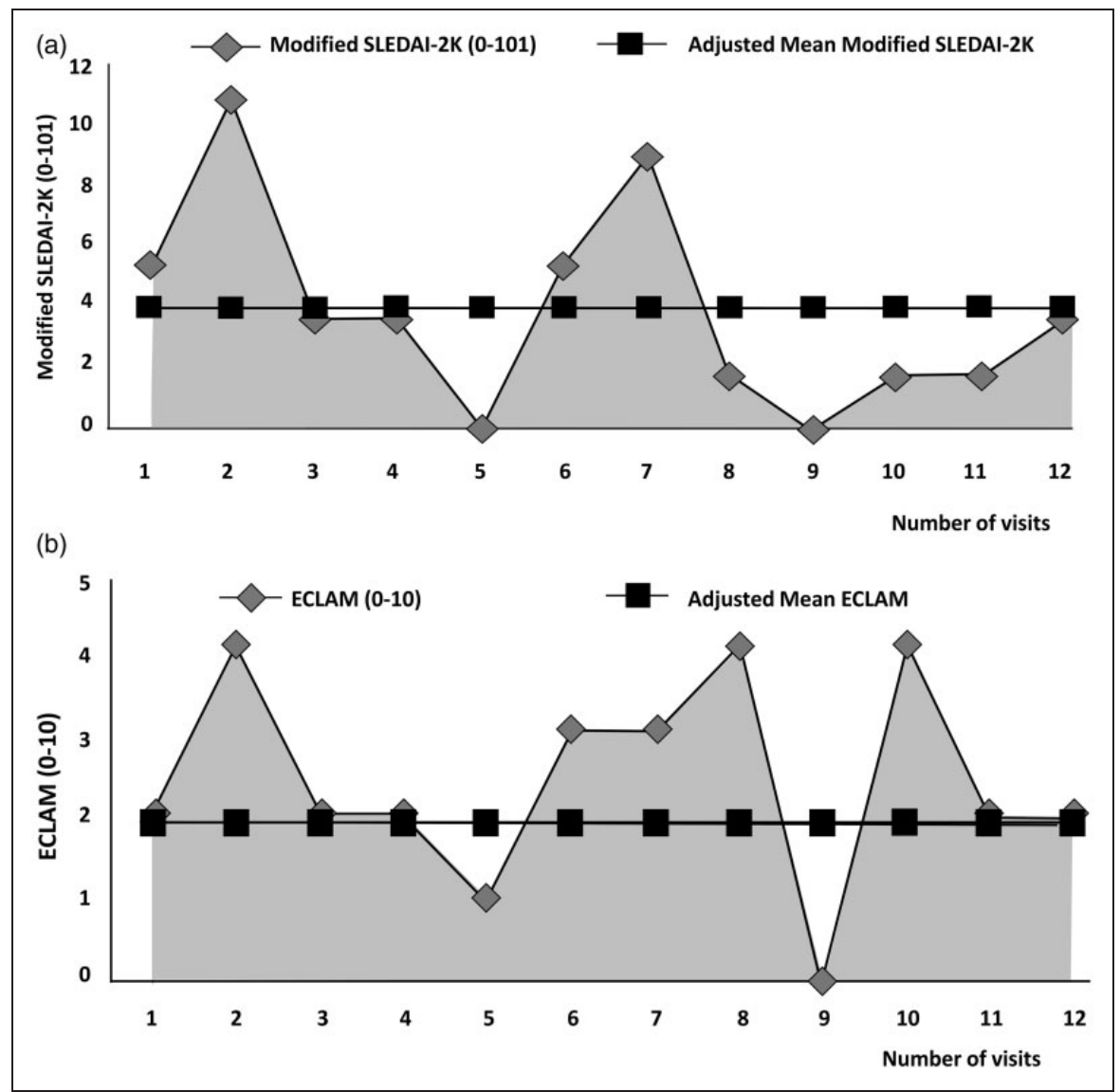

Figure 1 Example of the visit-to-visit disease activity scores for the Modified SLEDAI-2K (a) and ECLAM (b) from one patient. The calculated area under the curve is represented in gray and adjusted means are represented by the straight line. 


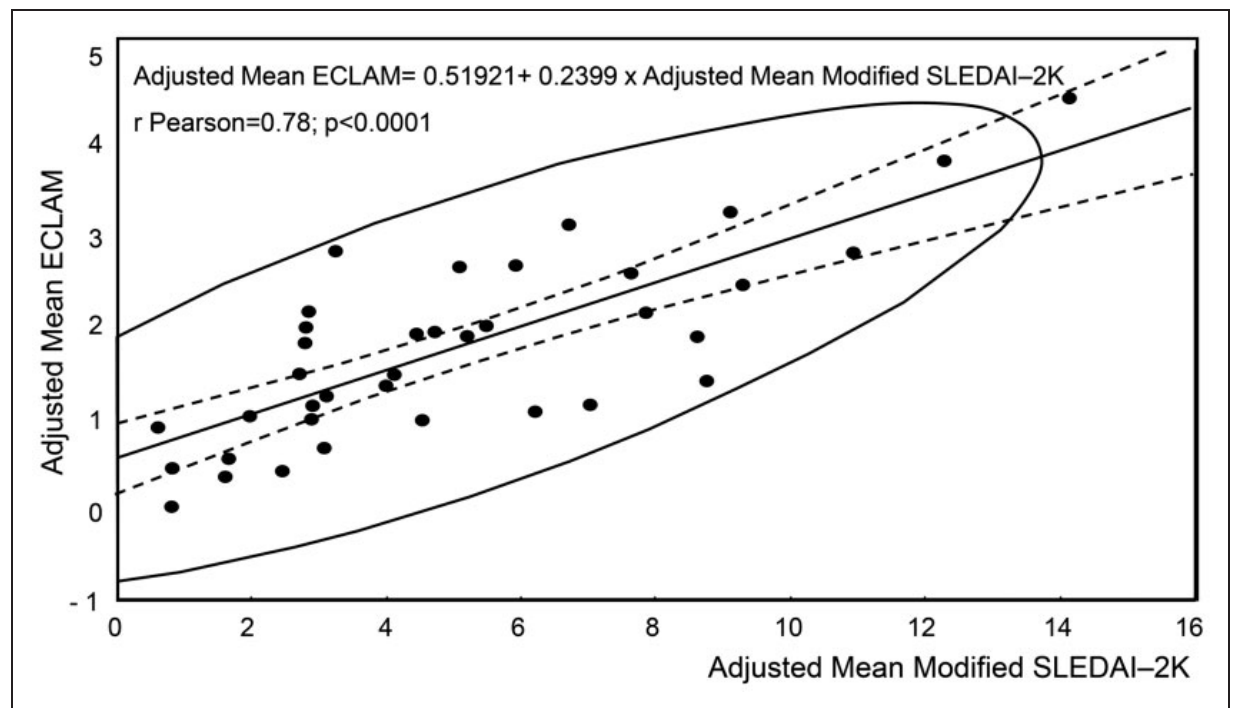

Figure 2 Correlation of the adjusted means of the Modified SLEDAI-2K and ECLAM by linear regression analysis.

refer to drugs used throughout the follow-up. Prednisone or prednisolone was used by 34 patients $(91.9 \%), 24(64.9 \%)$ with prednisone or prednisolone in combination with methylprednisolone pulse therapy; hydroxychloroquine was used by 33 patients $(89.2 \%)$, and immunosuppressive treatment was used by 20 patients $(54.1 \%)$, with the following distribution: intravenous cyclophosphamide $(40.5 \%)$, azathioprine $(40.5 \%)$, mycophenolate $(10.8 \%)$, and methotrexate $(5.4 \%)$. A total of 781 clinical visits, ranging from four to 59 (median 18) visits for each patient, were reviewed. A detailed report of clinical and demographic characteristics of the series has been reported previously. ${ }^{6}$

\section{Disease activity and damage assessment}

Modified SLEDAI-2K mean (SD) was 13.7 (9.2) and ECLAM mean (SD) was 4.9 (2.8) by the time of diagnosis. Their respective adjusted means from diagnosis to last follow-up visit were 5.1 (3.3) and 1.7 (1.0).

Twenty patients (62.5\%) accrued damage (Ped-SDI score $\geq 1$ ), with median 1 (IQR 0-2; range $0-8)$, considering only those with six months or longer follow-up $(n=32)$. Ten patients $(31.3 \%)$ had growth failure, eight $(25 \%)$ neuropsychiatric damage, four $(12.5 \%)$ ocular damage, four $(12.5 \%)$ musculoskeletal damage, and three $(9.4 \%)$ renal damage.

\section{Correlation between disease activity and damage indices}

Simple regression analysis resulted in high correlation between the adjusted means of the Modified
SLEDAI-2K and ECLAM, with $R^{2}=0.61$, Pearson $r=0.78$ and $p<0.001$. The linear equation for the model was (ECLAM adjusted mean $=(0.51921$ $+0.2399) \times$ Modified SLEDAI-2K adjusted mean), and the scatter plot for this model is presented in Figure 2.

Similarly, high correlation between both disease activity indices was found at disease onset and throughout disease course, with $r_{\mathrm{s}}>0.7$ and $p<0.001$, whereas only a moderate correlation was found between each disease activity index and the damage index, both at disease onset and throughout disease course, with $p<0.05$ for all correlations, except for the Modified SLEDAI-2K at disease onset and Ped-SDI (Table 1).

\section{Discriminant analysis}

The ROC curve for the Modified SLEDAI-2K and ECLAM adjusted means, considering 70\% sensitivity and $77 \%$ specificity for the presence of damage, resulted in both indices with similar performance discriminating damage, with AUC of 0.74 and 0.73 and cut-off points of 3.62 and 1.28 for Modified SLEDAI-2K and ECLAM, respectively.

\section{Discussion}

The correlation between activity and damage in a JSLE series retrospectively scoring the Modified SLEDAI-2K and ECLAM for activity, and PedSDI for damage, was evaluated. The retrospective scoring approach for activity and damage was as reliable as previous studies. ${ }^{6,10,11,13}$ Similarly, the 
Table 1 Spearman's rank correlation between disease activity and disease damage indices, at disease presentation and during disease course of a JSLE series

\begin{tabular}{ll}
\hline $\begin{array}{l}\text { At disease } \\
\text { presentation }\end{array}$ & $\begin{array}{l}\text { During } \\
\text { disease course } \\
\text { (adjusted means) }\end{array}$ \\
\hline $\begin{array}{l}\text { Modified SLEDAI-2K and ECLAM } \\
0.74\end{array}$ & 0.73 \\
$\quad p<0.001$ & $p<0.001$ \\
$\quad n=37$ & $n=37$ \\
Modified SLEDAI-2K and Ped-SDI & \\
0.32 & 0.51 \\
$p=0.07$ & $p=0.003$ \\
$n=32$ & $n=32$ \\
ECLAM and Ped-SDI & \\
0.39 & 0.48 \\
$p=0.03$ & $p=0.006$ \\
$n=32$ & $n=32$ \\
\hline
\end{tabular}

use of adjusted mean values for disease activity indices, as "summary measures" during disease course was also reliable. This association was strongly associated with damage and mortality in adults. ${ }^{12,14}$ Besides, these choices considered only quantitative global scales.

To our knowledge, the Modified SLEDAI-2K has not been used previously in the assessment of disease activity in JSLE. We chose to explore Modified SLEDAI-2K because of its practical suppression of complement and anti-dsDNA measures, which makes it more feasible for daily practice. There are literature reports of equivalence among the most commonly-used disease activity indices. ${ }^{2,4,5}$ However, ECLAM may be more responsive ${ }^{5}$ and less skewed than the SLEDAI. ${ }^{2}$ Our results confirm the previous findings by the linear correlation of Modified SLEDAI-2K with ECLAM.

The similarity in the correlation coefficients found by Pearson and Spearman tests confirms the linear relationship between Modified SLEDAI-2K and ECLAM, pointing to high correlation. Only moderate correlation between the SLEDAI and ECLAM was found in a previous paper. ${ }^{15}$ This could indicate a better performance when using the Modified SLEDAI-2K and the adjusted mean values of both activity indices.

The relationship between disease activity and damage accrual in JSLE has been reported previ-

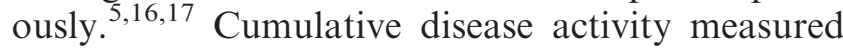
by SLEDAI and ECLAM were found to predict damage. ${ }^{5,18}$ In addition to the high mutual correlation, both activity indices also correlated significantly with the damage index. We observed also similar discriminating properties in both activity indices related to the damage index.

However, these results have the limitation of single center, small series, retrospective scores, and the possibility of under-estimated activity and damage measures due to missing clinical data of a multi-organ disease. Also, it was not possible to evaluate the treatment received over time in relation to activity scores and damage accrual. A more detailed analysis of treatment over time was hampered by retrospective data collection along a time frame with a major treatment shifts paradigm, including immunosuppressive treatment and steroid sparing strategies. More recent guidelines reflecting this paradigm have been adopted for the last years of the study time-frame. ${ }^{19}$

Nevertheless, we observed linear correlation between Modified SLEDAI-2K and ECLAM by two different analyses. It is also important to consider that the choice of the activity index should rely on the purpose of the study, level of training, and investigator preference. ${ }^{2}$

In spite of all acknowledged limitations, this exploratory analysis indicated both tools are equally useful for longitudinal estimates of JSLE activity conducted during daily practice.

\section{Declaration of Conflicting Interests}

The author(s) declared no potential conflicts of interest with respect to the research, authorship, and/or publication of this article.

\section{Funding}

The author(s) disclosed receipt of the following financial support for the research, authorship, and/ or publication of this article: JO Sato has received funding from the São Paulo Research Foundation (FAPESP; grant number 2014/07659-0) and UNESP Post-Graduate Public Health Program: PhD thesis (2009-2013). JE Corrente is a CNPQ scholar (304801/2014-3). C Saad-Magalhães is a CNPQ scholar (301644-2010, 301479/2015).

\section{References}

1 Brunner HI, Ravelli A. Developing outcome measures for paediatric rheumatic diseases. Best Pract Res Clin Rh 2009; 23: 609-624.

2 Lattanzi B, Consolaro A, Solari N, Ruperto N, Martini A, Ravelli A. Measures of disease activity and damage in pediatric systemic lupus erythematosus: British Isles Lupus Assessment Group (BILAG), European Consensus Lupus Activity Measurement 
(ECLAM), Systemic Lupus Activity Measure (SLAM), Systemic Lupus Erythematosus Disease Activity Index (SLEDAI), Physician's Global Assessment of Disease Activity (MD Global), and Systemic Lupus International Collaborating Clinics/American College of Rheumatology Damage Index (SLICC/ACR DI; SDI). Arthrit Care Res 2011; 63: S112-117.

3 Uribe AG, Vila LM, McGwin G Jr, Sanchez ML, Reveille JD, Alarcon GS. The Systemic Lupus Activity Measure-revised, the Mexican Systemic Lupus Erythematosus Disease Activity Index (SLEDAI), and a modified SLEDAI-2K are adequate instruments to measure disease activity in systemic lupus erythematosus. J Rheumatol 2004; 31: 1934-1940.

4 Brunner HI, Feldman BM, Bombardier C, Silverman ED. Sensitivity of the Systemic Lupus Erythematosus Disease Activity Index, British Isles Lupus Assessment Group Index, and Systemic Lupus Activity Measure in the evaluation of clinical change in childhood-onset systemic lupus erythematosus. Arthritis Rheum 1999; 42: 1354-1360.

5 Brunner HI, Silverman ED, Bombardier C, Feldman BM. European Consensus Lupus Activity Measurement is sensitive to change in disease activity in childhood-onset systemic lupus erythematosus. Arthritis Rheum 2003; 49: 335-341.

6 Sato JO, Corrente JE, Saad-Magalhães C. Chronic active disease pattern predicts early damage in juvenile systemic lupus erythematosus. Lupus 2015; 24: 1421-1428.

7 Gutierrez-Suarez R, Ruperto N, Gastaldi R, et al. A proposal for a pediatric version of the Systemic Lupus International Collaborating Clinics/American College of Rheumatology Damage Index based on the analysis of 1,015 patients with juvenile-onset systemic lupus erythematosus. Arthritis Rheum 2006; 54: 2989-2996.

8 Hochberg MC. Updating the American College of Rheumatology revised criteria for the classification of systemic lupus erythematosus. Arthritis Rheum 1997; 40: 1725.

9 Vitali C, Bencivelli W, Isenberg DA, et al. Disease activity in systemic lupus erythematosus: report of the Consensus Study Group of the European Workshop for Rheumatology Research. II. Identification of the variables indicative of disease activity and their use in the development of an activity score. The European
Consensus Study Group for Disease Activity in SLE. Clin Exp Rheumatol 1992; 10: 541-547.

10 Fitzgerald JD, Grossman JM. Validity and reliability of retrospective assessment of disease activity and flare in observational cohorts of lupus patients. Lupus 1999; 8: 638-644.

11 Mosca M, Bencivelli W, Vitali C, Carrai P, Neri R, Bombardieri S. The validity of the ECLAM index for the retrospective evaluation of disease activity in systemic lupus erythematosus. Lupus 2000; 9: 445-450.

12 Ibanez D, Urowitz MB, Gladman DD. Summarizing disease features over time: I. Adjusted mean SLEDAI derivation and application to an index of disease activity in lupus. J Rheumatol 2003; 30: 1977-1982.

13 Bernatsky S, Clarke A, Abrahamowicz M, Neville C, Karp I, Pineau CA. A comparison of prospective and retrospective evaluations of the Systemic Lupus International Collaborating Clinics/ American College of Rheumatology Damage Index for systemic lupus erythematosus. J Rheumatol 2005; 32: 820-823.

14 Ibanez D, Gladman DD, Urowitz MB. Adjusted mean Systemic Lupus Erythematosus Disease Activity Index-2K is a predictor of outcome in SLE. $J$ Rheumatol 2005; 32: 824-827.

15 Meiorin S, Pistorio A, Ravelli A, et al. Validation of the Childhood Health Assessment Questionnaire in active juvenile systemic lupus erythematosus. Arthritis Rheum 2008; 59: 1112-1119.

16 Brunner HI, Silverman ED, To T, Bombardier C, Feldman BM. Risk factors for damage in childhood-onset systemic lupus erythematosus: cumulative disease activity and medication use predict disease damage. Arthritis Rheum 2002; 46: 436-444.

17 Bandeira M, Buratti S, Bartoli M, et al. Relationship between damage accrual, disease flares and cumulative drug therapies in juvenile-onset systemic lupus erythematosus. Lupus 2006; 15: $515-520$.

18 Ravelli A, Duarte-Salazar C, Buratti S, et al. Assessment of damage in juvenile-onset systemic lupus erythematosus: a multicenter cohort study. Arthritis Rheum 2003; 49: 501-507.

19 Hollander MC, Sage JM, Greenler AJ, et al. International consensus for provision of quality driven care in childhood onset Systemic Lupus Erythematosus. Arthrit Care Res 2013; 65: 1416-1423. 